\title{
Interférences
}

Ars scribendi

5 | 2009

Historia / Persona

\section{Cesare: commentarii, historiae, vitae}

\section{Giuseppe Zecchini}

\section{OpenEdition}

\section{Journals}

\section{Edizione digitale}

URL: http://journals.openedition.org/interferences/909

DOI: 10.4000/interferences.909

ISSN: $1777-5485$

\section{Editore}

HiSoMA - Histoire et sources des Mondes antiques

\section{Edizione cartacea}

Data di pubblicazione: 1 gennaio 2009

ISSN: 1777-5485

\section{Notizia bibliografica digitale}

Giuseppe Zecchini, « Cesare: commentarii, historiae, vitae », Interférences [En ligne], 5 | 2009, mis en ligne le 11 décembre 2014, consulté le 15 septembre 2020. URL : http://journals.openedition.org/ interferences/909; DOI : https://doi.org/10.4000/interferences.909

Questo documento è stato generato automaticamente il 15 settembre 2020.

Tous droits réservés 


\title{
Cesare: commentarii, historiae, vitae
}

\author{
Giuseppe Zecchini
}

1 Come sempre, in principio ci fu Silla. E' noto che egli fu modello a Cesare per tanti aspetti dell'azione politica, dall'uso spregiudicato di un esercito ormai politicizzato alla marcia su Roma, dalla dittatura (sia pure a tempo indeterminato, e non perpetua) all'immissione dei neocittadini italici in tutte le tribù; così, anche in campo storiografico è difficile concepire la genesi dei commentarii di Cesare senza il precedente sillano.

2 Certo, altri precedenti si possono indicare, risalendo lungo la storia della (auto)biografia romana. Gli elogi gentilizi come quelli degli Scipioni o di M.Claudio Marcello (se non addirittura quello di L.Papirio Cursore conservatoci da Livio) ${ }^{1} \mathrm{ci}$ riportano a testi scritti conservati negli archivi di famiglia almeno dal III secolo ${ }^{2}$; col I secolo il genere fu aperto anche alle donne e Cesare stesso vi aderì con gli elogi funebri della zia Giulia e della moglie Cornelia ${ }^{3}$. Questa tradizione genuinamente romana si vide affiancata dall'epistola memoriale di matrice ellenistica durante l'età delle conquiste transmarine e in concomitanza con la nascita della storiografia a Roma; non a caso sono i Cornelii Scipioni, la più ellenizzata delle gentes, a utilizzare per primi questo strumento di valorizzazione delle proprie res gestae e di diffusione della loro conoscenza in ambito internazionale (come già aveva fatto un altro grande condottiero ellenizzante, Annibale) $)^{4}$ : così P.Cornelio Scipione l'Africano in una lettera a Filippo V, così P.Cornelio Scipione Nasica in una lettera a Massinissa, così C.Sempronio Gracco, figlio di una Cornelia, in una lettera a M.Pomponio a proposito del proprio padre e del proprio fratello ${ }^{5}$.

3 E' già stato giustamente osservato ${ }^{6}$ che nell'età postgraccana, agli inizi del I secolo, la crisi della repubblica nobiliare e dell'egualitarismo tra i suoi componenti creò $i$ presupposti per l'emergere di singole personalità militanti in campi opposti nell'agone politico e quindi per l'esigenza di affidare a testimonianze (auto)biografiche l'apologia delle proprie azioni (o di quelle dei leaders della propria pars) di fronte alle accuse degli avversari. Di nuovo furono gli ottimati più ellenizzati a sfruttare per primi questo strumento letterario e a imprimergli un vigore, che non aveva riscontri nella cultura ellenistica: mentre Q.Lutazio Catulo conservò la forma epistolare al suo Liber de 
consulatu et de rebus gestis suis per tramandare appunto le sue res gestae contro i Cimbri e in rivalità con Mario nel $101^{7}$, M.Emilio Scauro e P.Rutilio Rufo scelsero la forma dell'autobiografia in prosa, molto ampia e quindi articolata in più libri (almeno tre e cinque rispettivamente) ${ }^{8}$; peraltro anche Catulo non si sottrasse all'esigenza di un impegno letterario, che, come richiesto dai tempi nuovi, andava ormai al di là dalla semplice testimonianza su fatti vissuti in prima persona: Cicerone ce ne attesta l' imitatio Xenophontis'; sappiamo inoltre che il Liber era dedicato al poeta A. Furio (Anziate?), forse per suggerire una rielaborazione in chiave epica, come fece Archia per Mario e Lucullo ${ }^{10}$.

4 Tuttavia è innegabile, ed è già stato riconosciuto ${ }^{11}$, che l'opera di Silla rappresenti un salto di qualità. Egli non scrisse né una lettera, né un'autobiografia, ma commentarii, non de vita sua, ma de rebus gestis (le testimonianze di Plutarco, Suetonio, Gellio e Prisciano sono concordi in tal senso) ${ }^{12}$; li articolò in ben ventidue libri, conferendo loro un'ampiezza pari alla gloria delle sue imprese: vi doveva narrare gli esordi nella vita pubblica, la guerra sociale e quella mitridatica, la guerra civile e forse anche la dittatura sino alla sua volontaria deposizione e alla restaurazione dell'ortodossia repubblicana in un tono certamente apologetico, ma non nel banale senso di giustificare il proprio operato dalle critiche degli avversari, bensì con l'intenzione di dare la propria chiave di lettura di quegli eventi, in cui aveva agito e trionfato come favorito degli dei e della Fortuna ${ }^{13}$; li dedicò al fidato Lucullo per una successiva rielaborazione in historiae ${ }^{14}$; come è noto, Lucullo non assolse questo compito, ma l'importanza e, direi, la paradigmaticità dei commentarii sillani all'interno della tradizione storiografica sulla sua epoca fu riconosciuta per altre vie: il liberto L.Cornelio Epicado avvertì l'esigenza di completarli, probabilmente fino alla morte, dopo che il suo padrone vi stava lavorando ancora quarantotto ore prima di spirare ${ }^{15}$; L.Cornelio Sisenna, il primo autore romano di historiae e comunque il più autorevole storico del suo tempo, se ne servì come fonte ${ }^{16}$; Cicerone nel 44 riconobbe loro con volontà polemica quella dignità di historia, che due anni prima aveva rabbiosamente negato ai commentarii di Cesare ${ }^{17}$.

5 Dopo Silla e dati i riconoscimenti ottenuti dai suoi commentarii, non era più possibile sottrarsi al confronto, anche letterario: esemplari sono le angosciate vicende di Cicerone a proposito del suo consolato nel 63 e della congiura di Catilina, con le sue composizioni poetiche, con la compilazione di un memoriale da fornire come base per un'opera storica e infine con la reiterata ricerca di un autore disposto a narrare le sue gesta in quell'occasione ${ }^{18}$.

6 Su questo imprescindibile sfondo sillano, su cui ho sentito il dovere di soffermarmi sin qui, noi dobbiamo leggere la produzione letteraria su e di Cesare.

7 Essa comincia, nel corso della guerra gallica, nella forma, in un certo senso già un po' superata, dei poemi storici, quello di Varrone Atacino sul Bellum Sequanicum, composto a celebrare la vittoria su Ariovisto nel 58, e quello di Furio Bibaculo sui Pragmatia belli Gallici, forse subito dopo Alesia, tra il 52 e il $50^{19}$; in realtà di questi poemi non sappiamo nulla oltre il titolo, ma sappiamo che lo stesso Catullo, quanto mai ambivalente nei suoi atteggiamenti verso Cesare, amico di suo padre, registrò subito con accenti ammirati, non ironici, lo sbarco in Britannia ${ }^{20}$ : se ci si riflette, la produzione poetica immediatamente suscitata dalle res gestae di Cesare in Gallia è davvero notevole, almeno per quantità. 
Contemporaneamente Cesare scrisse di persona i commentarii su tali imprese, il Bellum Gallicum nel 51 (non credo a una composizione anno per anno, ma a una composizione unitaria $^{21}$, quando Cesare ebbe acquisito la certezza della vittoria e quindi di una conquista meritevole di commentarii), il Bellum civile, incompiuto, nel 46. L'evidente imitatio Xenophontis nell'adozione della III persona può ricordarci Catulo, ma tutto il resto rinvia a Silla: innanzitutto l'ampiezza della narrazione, dieci libri, circa la metà di quelli sillani; poi, la scelta degli argomenti, non solo un bellum externum, ma anche quello civile, proprio come in Silla quest'ultimo seguiva a quello mitridatico; ancora, quella déformation historique (per citare il titolo di un celebre saggio di M. Rambaud) ${ }^{22}$, che ha ormai sostituito come cifra dei commentarii la stravagante idea di un' 'obiettività' cesariana: essa è la felice definizione moderna di quella volontà apologetica, che, come si è visto, era già appartenuta a Silla; Cicerone la smascherava nel Brutus, laddove l'oppositore politico del dittatore manifestava tutta la sua preoccupazione per il rischio che la versione cesariana degli eventi si affermasse come l'unica, anche grazie all'indubbio fascino della sua prosa ${ }^{23}$; infine la fortuna postuma, cioè i rapporti con la successiva produzione storiografica, che richiede un discorso più complesso.

9 Non voglio qui addentrarmi nelle complesse vicende della genesi del corpus Caesarianum. Mi limito a ricordare innanzitutto che l'unico precedente della continuatio di un commentarius (che, in quanto tale, non aveva la dignità di un'opera di historia e non era quindi suscettibile di continuatio) era proprio il completamento dei commentarii di Silla da parte del suo liberto Epicado; certo, per Cesare la situazione era più complessa, perché egli era stato ucciso all'improvviso e non aveva potuto lavorare con calma alla stesura delle sue memorie fino a pochi giorni prima della sua morte: non si trattava quindi di aggiungere la sola conclusione a un testo pressoché ultimato, ma di riempire lacune molto ampie, e tuttavia l'iniziativa di Epicado valeva come non trascurabile exemplum.

Quattro (di fatto, come si vedrà, cinque) nuovi libri furono aggiunti ai dieci originari di Cesare stesso per completare la narrazione delle sue res gestae; la famosa lettera scritta da Irzio a Balbo come prefazione all'VIII libro del Bellum Gallicum è stata di recente vista come un falso retorico tardoantico e proprio l'VIII libro e i primi capitoli del BAl sono stati attribuiti a Cesare stesso, che li avrebbe lasciati incompiuti e inediti (come l'VIII libro di Tucidide) ${ }^{24}$, ma, anche se si accettassero queste nuove e non necessarie ipotesi, resta il fatto che o Cesare stesso o gli amici Caesaris subito dopo la morte del dittatore si posero il problema della tradizione delle sue res gestae: esse non erano complete, c'era un'evidente lacuna tra Bellum Gallicum e Bellum civile e, secondo uno dei più colti ed autorevoli tra loro, C.Asinio Pollione, Cesare stesso avrebbe riscritto i suoi commentarii, se ne avesse avuto il tempo ${ }^{25}$; prima della metà del 43 , dunque nell'anno immediatamente successivo al cesaricidio, qualcosa si fece, se dobbiamo ad A. Irzio, morto appunto nel 43 a Modena, la composizione o l'edizione dell'VIII libro del Bellum Gallicum e la composizione almeno della prima parte del Bellum Alexandrinum, che narra la guerra in Egitto e fungeva da necessario completamento del III libro del Bellum civile, interrotto in modo assai brusco. La seconda parte del Bellum Alexandrinum, che è in realtà un Bellum Ponticum, che è attaccato al precedente in modo artificioso e che costituisce in effetti un commentarius a se stante, forse di Irzio, forse di autore ignoto, il Bellum Africum, di un legato di Cesare, forse L.Munazio Planco ${ }^{26}$, infine il Bellum Hispaniense, di un eques Romanus ex provinciis, forse L.Vibio Pacieco ${ }^{27}$, sono di provenienza diversa tra loro e non furono originariamente scritti allo scopo di 
completare Cesare, ma furono raccolti e messi insieme a tale scopo: soprattutto l'evidente anomalia rappresentata dal Bellum Hispaniense, molto diverso dagli altri commentarii per prospettiva etnico-sociale e per stile, rivela la fretta di fornire un quadro delle res gestae di Cesare, che da una prospettiva comunque cesariana costituisse la versione di riferimento per qualsiasi storico successivo: il timore espresso da Cicerone nel Brutus non era dunque infondato, ma era la consapevole presa di coscienza che Cesare e i suoi avevano la volontà di manipolare la storia e la stavano traducendo in un progetto assai concreto.

11 I risultati di questo sforzo editoriale sono noti; anche se accettiamo l'ipotesi, da me sostenuta, ma non da tutti condivisa, che Cassio Dione ci conservi una versione storiografica alternativa ai commentarii e per di più di tendenza opposta ${ }^{28}$, nondimeno è incontestabile che la versione cesariana delle res gestae di Cesare abbia sbaragliato ogni concorrenza: delle guerre di Cesare i posteri leggono ciò che i cesariani ci hanno voluto tramandare.

12 Tuttavia quest'operazione non era sufficiente. Infatti i commentarii erano limitati alle imprese militari e lasciavano, per così dire, scoperte la fase iniziale e quella finale della vita pubblica di Cesare, che si erano svolte a Roma e appartenevano alla politica. Per inserire Cesare nella storia interna di Roma ci voleva uno storico, come era stato Sisenna per Silla, e gli amici Caesaris ne avevano a disposizione ben due, di altissimo livello, C.Sallustio Crispo e C.Asinio Pollione. Coadiuvati dal medesimo consulente, il liberto C.Ateio Pretestato, essi intrapresero la stesura di due Historiae, la prima dal 78, cioè dalla fine di Sisenna, al 60 , la seconda dal 60 al 44/42 29 ; esse avrebbero dovuto quindi saldarsi al 60, l'anno del cosiddetto I triumvirato, che M.Terenzio Varrone aveva denunciato in tono allarmato nel suo Tricaranus ${ }^{30} \mathrm{e}$ che costituiva invece, in prospettiva opposta, l'anno dell'elezione di Cesare a console e l'inizio della resa dei conti con la nobiltà; il centro 'ideale' delle Historiae sallustiane era già stato anticipato nel Catilina, quando Cesare esordì come neoeletto pontefice massimo, sollevando la questione della liceità morale del S.C. ultimum, una delle chiavi di volta dell' 'ideologia' popularis ${ }^{31}$; il centro 'ideale' delle Historiae di Pollione era invece la denuncia del cesaricidio interpretato non certo come un tirannicidio o comunque un atto politico, ma come un sacrilegio, in quanto Cesare era appunto pontefice massimo, e quindi come una profanazione di natura religiosa del tutto inaccettabile ${ }^{32}$.

13 Anche sul piano storiografico la formidabile coppia 'Sallustio-Pollione' grazie alla sopravvivenza del Catilina e di vasto materiale pollioniano almeno in Velleio, in Plutarco e soprattutto in Appiano ha esercitato un influsso non trascurabile, se pur qui non esclusivo ed anzi bilanciato da versioni di segno opposto.

14 La personalità di Cesare non si esauriva però nelle sue straordinarie performances politico-militari, vi era anche un lato privato, a partire da una giovinezza oscura, sulla quale circolavano i più svariati e improbabili aneddoti, non tutti certo di segno positivo (basti pensare ai suoi presunti rapporti con Nicomede di Bitinia) ${ }^{33}$; vi era il Cesare intimo, quello dei pensieri e delle confidenze più riposte; vi erano episodi spesso trascurabili sul piano della 'grande storia', ma capaci di rivelarne l'indole e il carattere: insomma, vi era tutto quel materiale, che lo storico tralasciava come 'minore', ma che un biografo avrebbe potuto valorizzare come particolarmente significativo.

15 C.Oppio si assunse questo compito ${ }^{34}$. Qui il confronto con Silla cessa, perché Silla non aveva avuto biografi: non sappiamo se Varrone gli dedicasse una delle sue Imagines, certamente Nepote lo dovette comprendere tra i duces romani nella corrispondente 
sezione delle sue Vitae, ma ora non ci interessano le ampie sillogi biografiche degli anni $30^{35}$, disordinato preludio alla sintesi augustea degli Elogia epigrafici nel Foro romano; qui non si tratta infatti di prendere in considerazione le origini della biografia romana in quanto forma parallela e, in qualche modo, sostitutiva della storiografia, ma di individuare i personaggi, a cui furono dedicate singole biografie nell'età della crisi della repubblica e della dissoluzione dell'ordinamento sociale nobiliare.

Allora, da questo più ristretto, ma anche più significativo punto di vista i dati a nostra disposizione sono pochi e chiari: Varrone compose in tre libri un'ampia biografia del suo 'campione', Pompeo, Q.Munazio Rufo scrisse una Vita di Catone, appunto Oppio si dedicò a un trittico di Vitae, quella di Scipione l'Africano maggiore, quella di Cassio e appunto quella di Cesare ${ }^{36}$.

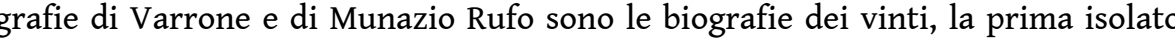
omaggio di un amico ${ }^{37}$, la seconda inserita nel già ricco filone della letteratura sull'Uticense, che tradusse dalla forma del libello e della laudatio in quella letterariamente più nobile e complessa propria appunto del genere biografico ${ }^{38}$ : sappiamo che almeno fino all'età di Nerone e alla biografia catoniana di Trasea Peto (ma anche al poema di Lucano) la sua fortuna fu notevole.

Le biografie di Oppio rispondono a un altro criterio. Esse ruotano intorno a quella dell'amico Cesare, ma sono consacrate anche al suo più prestigioso e incontestato modello e a uno dei capi della congiura contro il dittatore. Riguardo a Scipione circolava un libello contemporaneo, inserito da Livio nella sua opera ${ }^{39}$, che lo presentava come colui che aveva rifiutato la dittatura perpetua, cioè come l'antiCesare; d'altra parte un legato di Cesare e politologo come L.Aurunculeio Cotta nel suo De re publica del 56/55 ca. aveva già stabilito un preciso parallelo tra l'altro grande Scipione, l'Emiliano, e Cesare stesso ${ }^{40}$, mentre è noto che sempre l'Emiliano assurge a protagonista del celebre finale del somnium Scipionis nel di poco successivo De re publica di Cicerone: i due Scipioni erano ormai patrimonio comune della memoria storica di Roma e non si poteva lasciarli alla parte avversa; perciò Oppio dovette rivendicare al 'suo' Cesare l'eredità ideale e politica del vincitore di Zama e sottolineare di quest'ultimo non tanto la correttezza istituzionale quanto il conflitto con l'ingrato senato, irrispettoso della sua dignitas, che in ultima analisi risaliva alla sua filiazione divina da Giove come quella di Cesare si collegava alla sua discendenza da Venere ${ }^{41}$. Riguardo a Cassio era opportuno ribadire il suo ruolo preponderante nella congiura per ridimensionare quello dell' 'idealista' Bruto, l'austero stoico imparentato con Catone: Bruto era il volto presentabile dei congiurati e rischiava di trasformarsi, come già l'Uticense, in un 'mito', quello del tirannicida e del liberatore; era meglio chiarire che le Idi di marzo erano state invece uno squallido affare di invidie e rancori personali, come quelli che avevano ispirato Cassio ${ }^{42}$.

19 Su questo sfondo si colloca la biografia di Cesare, quella che conosciamo meglio grazie ai frammenti superstiti e che godette di discreta fortuna nell'antichità, almeno in Gallia, dove forse era ancora letta nel v secolo ${ }^{43}$. Non credo casuale che i frammenti conservati da Plutarco e da Suetonio, cioè dai successivi biografi di Cesare, riguardino il Cesare privato: Plutarco riferisce dell'infelice destino del coltissimo Q.Valerio Sorano, fatto uccidere da Pompeo, e, nel contesto, cita Oppio riguardo alla sua abitudine di classificare molti personaggi come 'amici' o 'nemici' di Cesare, aggiungendo che bisogna però servirsi con cautela di tali categorie; ancora Plutarco usa Oppio come testimone delle straordinarie doti di Cesare come cavaliere; Suetonio invece lo cita per 
celebrare l'indifferenza di Cesare verso il cibo e la sua delicatezza d'animo nei confronti di chi lo ospitava; infine Plutarco e Suetonio ricordano il medesimo aneddoto concernente la generosità di Cesare verso Oppio stesso (e dunque derivato molto probabilmente dalla sua biografia), quando questi era malato ${ }^{44}$. Come si vede, i temi sono i rapporti personali di amicizia e inimicizia, le abilità fisiche di Cesare, le sue virtù come l'affabilità e l'attenzione nel soccorrere gli altri; si badi che queste due ultime qualità, la facilitas e il sublevare, si ritrovano nel famoso 'ritratto' di Cesare, che Sallustio delinea nel Catilina del $43^{45}$, subito all'indomani del cesaricidio: esse erano dunque elementi costitutivi di un'immagine ideale di Cesare, che i suoi amici intendevano tramandare; a questa immagine di uomo completo di ogni virtù fisica e morale, di Idealtypus del vir Romanus, che Cesare doveva incarnare, era senza dubbio consacrata la biografia di Oppio.

Credo di aver così delineato $\mathrm{i}$ cinque stadi successivi della produzione letteraria $\mathrm{e}$ storiografica, che contribuì a costruire, se così posso esprimermi, l'immagine cesariana di Cesare: prima i poemi epico-storici, celebrativi di eventi appena accaduti, poi la riflessione storiografica di Cesare affidata a commentarii, di per sé solo Materialien zur Geschichte in attesa di ulteriore elaborazione altrui, in realtà già così perfezionati sul piano sia contenutistico, sia formale da ambire ad essere 'storia' tout court, poi il loro completamento attraverso altri commentarii in grado di costituire un corpus completo delle res gestae militari di Cesare, poi le 'Storie' di Sallustio e Pollione, ulteriore completamento sul versante interno e politico dell'attività pubblica di Cesare e del suo significato all'interno della crisi della res publica, infine la biografia di Oppio, che poteva occuparsi anche del Cesare privato, come peraltro richiedeva il genere letterario prescelto.

21 Gli ultimi tre stadi sono tutti posteriori alla morte di Cesare e furono promossi dai suoi amici: essi sono da situare negli anni 30 e sono quindi coevi tra loro; corrispondono a una fortissima, impressionante volontà di trasmissione della memoria storica, che per coerenza e complessità di disegno non ha eguali nella storiografia antica. Non ci fu dalla parte opposta una replica di pari dimensioni, soprattutto sui tempi brevi: forse si dovette attendere Livio per avere una visione 'pompeiana' della guerra civile del 49/4546; nell'immediato ci si limitò a lavorare invece intorno al 'mito' di Catone come unica alternativa a Cesare.

Quando Sallustio scrisse nel Catilina che dalla dissoluzione della repubblica emergevano e si salvavano solo due uomini, Cesare e Catone, egli emetteva un giudizio storico e, senza saperlo, anche una profezia storiografica: alla biografia cesariana di Oppio si contrappose quella catoniana di Munazio Rufo. Cicerone, l'optimus consul detestato da Sallustio ${ }^{47}$, non ebbe biografi, forse anche perché, come è noto, aveva anteposto le istituzioni all'amicitia ${ }^{48}$.

A differenza di Cesare e come Silla, Augusto ebbe lunga vita e morì nel proprio letto; trovò il tempo di scrivere ben due autobiografie, a distanza di vent'anni una dall'altra, $\mathrm{e}$ di promuovere due biografie, composte da un suo amico molto stretto, Nicola di Damasco, e da un suo liberto, Giulio Marato ${ }^{49}$ : ormai era il princeps e di fatto solo lui poteva scrivere o far scrivere di se stesso. Alla prima opzione si attennero, a partire da Tiberio, parecchi tra i successivi imperatori e fiorì quindi l'autobiografia imperiale ${ }^{50}$; quanto alla biografia di uomini illustri, così vivace durante la crisi della repubblica, essa fu per il momento accantonata: sotto il principato si tornava ad una nuova 
omologazione, da cui non era bene che emergessero personalità tali da far ombra al sovrano.

\section{BIBLIOGRAFIA}

BAIER T. 2005, «Autobiographie in der späten römischen Republik», in M. Reichel (ed.), Antike Autobiographien, Köln, p.123-142.

BEHR H. 1993, Die Selbstdarstellung Sullas: ein aristokratischer Politiker zwischen persönlichem Führungsanspruch und Standessolidarität, Europäische Hochschulschriften. Reihe 3, Geschichte und ihre Hilfswissenschaften 539, Frankfurt am Main.

BLÄNSDORF J. (ed.) 1995, Fragmenta poetarum latinorum epicorum et lyricorum: praeter ennium et lucilium, Bibliotheca scriptorum Graecorum et Romanorum Teubneriana, Stutgardiae.

BRUGNOLI G. 1993, «Caesar grammaticus», in D. Poli (ed.), La cultura in Cesare: atti del convegno internazionale di studi, [Università di Macerata], Macerata-Matelica, 30 aprile-4 maggio 1990, II, Quaderni linguistici e filologici 5, Roma, p.585-597.

BRUNT P.A. 1965, «Amicitia in the late Roman Republic», PCPhS 11, p.1-20.

CALABI I. 1950, «I commentarii di Silla come fonte storica», RAL ser.8, v.3, fasc.5., p.247-302.

CANFORA L. 1970, «Cesare continuato», Belfagor 25, p.419-429.

CANFORA L. 1993, «La lettera a Balbo e la formazione della raccolta cesariana», ASNP 23, p.79-103.

CESA M. 2007, «Riflessioni sulla composizione del De bello Gallico», in P. Desideri, M. Moggi, M. Pani (edd.), Antidoron: Studi in onore di Barbara Scardigli Forster, Pisa, p.37-50.

DOBESCH G. 2006, «Varro Atacinus und sein Bellum Sequanicum», in Philia: Festschrift für Gerhard Wirth zum 80. Geburtstag am 9. Dezember 2006, Historia antiqua Galatiensis 2, Galati, p.123-174.

FERRARY J.L. 1997, «Optimates et populares : le problème du rôle de l'idéologie dans la politique», in H. Bruhns, J.-M. David, W. Nippel (edd.), La fin de la République romaine = Die späte Römische Republik: un débat franco-allemand d'histoire et d'historiographie, Coll. de l'École française de Rome 235, Rome, p.221-231.

GEIGER J. 1985, Cornelius Nepos and Ancient Political Biography, Historia. Einzelschriften 47, Stuttgart. Granarolo J. 1958, «Catulle et César», Annales de la Faculté de Lettres d’Aix 32, p.53-73.

JEHNE M. 1997, Caesar, Becksche Reihe 2044, München.

KIERDORF W. 1980, Laudatio funebris: Interpretationen und Untersuchungen zur Entwicklung der römischen Leichenrede, Beiträge zur klassischen Philologie 106, Meisenheim am Glan.

KRUSCHWITZ P. 1998, «Die Datierung der Scipionenelogien CLE 6 und 7», ZPE 122, p.273-285.

KRUSCHWITZ P. 2002, Carmina Saturnia Epigraphica: Einleitung, Text und Kommentar zu den Saturnischen Versinschriften, Hermes. Einzelschriften 84, Stuttgart.

LEWIS R.G. 1991, «Sulla's Autobiography: Scope and Economy», Athenaeum 69, p.509-519. 
LORETO L. (ed.) 2001, Pseudo-Cesare. La lunga guerra civile: Alessandria-Africa-Spagna, BUR. Classici Greci e Latini L1342, Milano.

MARSHALl A. 1993, «Atticus and the genealogies», Latomus 52, p.307-317.

MINEO B. 2009, «Le 'pompéianisme' de Tite-Live», in O. Devillers, J. Meyers (edd.), Pouvoirs des hommes, pouvoir des mots, des Gracques à Trajan : hommages au professeur Paul Marius Martin, Bibliothèque d'études classiques 54, Louvain - Paris - Walpole (Mass.), p.277-289.

NARDUCCI E. 1989, « Le risonanze del potere », in G. Cavallo, P. Fedeli, A. Giardina (edd.), Lo spazio letterario di Roma antica. II, La circolazione del testo, Roma, p.533-577.

PALLAVISINI A. 1974, «Il capitolo 22 del Bellum Africum e la propaganda augustea», in M. Sordi (ed.), Contributi dell'Istituto di Storia Antica, II, Milano, p. 107-114.

PASCUCCI G. 1975, «I commentarii di Silla», in S. Boldrini et al. (edd.), Atti del Convegno gli storiografi latini tramandati in framenti, Urbino, 9-11 maggio 1974, Studi urbinati di storia, filosofia e letteratura 49, Urbino, p.282-296.

PITTIA S. 2009, «L'empereur romain historien», in G. Zecchini (ed.), Lo storico antico: mestieri e figure sociali, atti del Convegno internazionale (Roma, 8-10 novembre 2007), Pragmateiai 17, Bari, p.65-94.

RAMBAUD M. 1953, L'art de la déformation historique dans les Commentaires de César, Annales de l'université de Lyon. Lettres 23, Paris [2 éd. 1966].

SALAMON G. 2009, «Cicéron entre histoire et biographie : propositions pour une relecture de la lettre de Cicéron à Lucceius (ad Fam. 5, 12)», Interférences. Ars scribendi 6 [in rete].

scholz P. 2003, «Sullas commentarii - eine literarische Rechtfertigung», in U. Eigler, U. Gotter, N. Luraghi et al. (edd.), Formen römischer Geschichtsschreibung von den Anfängen bis Livius: Gattungen - Autoren - Kontexte, Darmstadt, p.172-195.

SCHOLz P. 2007, «Autobiographien hellenistischer Herrscher und republikanischer nobiles - Ein Unterschied der Volksindividualität?», in M. Erler, S. Schorn (edd.), Die griechische Biographie in hellenistischer Zeit: Akten des internationalen Kongresses vom 26.-29. Juli 2006 in Würzburg, Beiträge zur Altertumskunde 245, Berlin - New York, p.385-406.

SONNABEND H. 2002, Geschichte der antiken Biographie: von Isokrates bis zur Historia Augusta, Stuttgart - Weimar.

STRASBURGER H. 1938, Caesars Eintritt in die Geschichte, München.

STRASBURGER H. 1968, Caesar im Urteil seiner Zeitgenossen, 2., durchgesehene und erg. und durch ein Nachwort erw. Aufl. Sonderausg., Libelli 158, Darmstadt.

STROCCHI F. 1996, «L'autore del Bellum Hispaniense», RIL 130, p.99-112.

TUPLIN C. 2000, «Nepos and the Origins of political Biography», in C. Deroux (ed.), Studies in Latin Literature and Roman History, X, Coll. Latomus 254, Bruxelles, p.124-161.

VALGIGLIO E. 1975, «L'autobiografia di Silla nelle biografie di Plutarco», in S. Boldrini et al. (edd.), Atti del Convegno gli storiografi latini tramandati in framenti, Urbino, 9-11 maggio 1974, Studi urbinati di storia, filosofia e letteratura 49, Urbino, p.245-281.

ZECCHINI G. 1978, Cassio Dione e la guerra gallica di Cesare, Pubblicazioni dell'Università cattolica del Sacro Cuore. Contributi. Serie terza. Scienze storiche 19, Milano.

ZECCHINI G. 1980, «La morte di Catone e l'opposizione intellettuale a Cesare e ad Augusto», Athenaeum 58, p.39-56. 
ZECCHINI G. 1982, «Asinio Pollione: dall'attività politica alla riflessione storiografica», in Aufstieg und Niedergang der römischen Welt, II, 30, 2, Berlin - New York, p.1265-1296.

ZECCHINI G. 1987, «I confini occidentali dell'impero romano: la Britannia da Cesare a Claudio», Contributi dell'Istituto di Storia Antica 13, p.250-271.

ZECCHINI G. 1995, « Sallustio, Lucullo e i tre schiavi di C.Giulio Cesare», Latomus 54, p.592-607.

ZECCHINI G. 1996, «Cicerone in Sallustio», in C. Stella, A. Valvo (edd.), Studi in onore di Albino Garzetti, Brescia, p.527-538.

ZECCHINI G. 2001, Cesare e il “mos maiorum”, Historia. Einzelschriften 151, Stuttgart.

ZECCHINI G. 2009, «Cesare : tirannicidio o sacrilegio?», in O. Devillers, J. Meyers (edd.), Pouvoirs des hommes, pouvoir des mots, des Gracques à Trajan : hommages au professeur Paul Marius Martin, Bibliothèque d'études classiques 54, Louvain - Paris - Walpole (Mass.), p.209-218.

zUCCHELli B. 1976, «L'enigma del Tpıkápavos : Varrone di fronte ai triumviri», in Atti del Congresso internazionale di studi varroniani: Rieti, settembre 1974, Rieti, p.509-526.

\section{NOTE}

1. ILLRP 309-312 (elogi degli Scipioni; i primi due, 309 e 310, datati tra il 270/230 e il 150 da Kruschwitz 1998; Kruschwitz 2002, p.32); Liv. 27,27,12-14 (Marcello); Liv. 9,16,11-19 (Cursore).

2. Sonnabend 2002, p.87.

3. La prima laudatio per una donna fu quella di Q.Lutazio Catulo per sua madre (Cic. De orat. 2,44); le laudationes di Cesare in Suet. DJ 6; sulla laudatio funebris in genere è fondamentale Kierdorf 1980.

4. La lettera di Annibale ai Rodii in Corn.Nep. Hann. 13,2.

5. Lettera di Scipione in Polyb. 10,2,1 ( $\mathrm{n}^{\circ} 232$ Jacoby = L Cugusi); lettera di Scipione Nasica in Plut. Aem.Paul. 15 e 17,8 (n'233 Jacoby = CIX Cugusi); lettera di Gracco in Plut. Ti.Gracch. 8 (CXXII Cugusi). I destinatari non romani delle prime due e la mancanza di diffusione della prima (Cic. De off. 3,4 ) escludono scopi apologetici ad uso interno, come vorrebbe Sonnabend 2002, p.89-90.

6. Scholz 2007, p.397-398. Cfr. anche Sonnabend 2002, p.93.

7. Il titolo completo in Cic. Brut. 35,132, il carattere epistolare in Front. 120,3-7 van den Hout (extant Catuli litterae...).

8. Sulle due opere cfr. da ultimo Sonnabend 2002, p.92-93 e Scholz 2007, p.392-395 e Baier 2005.

9. Cic. Brut. 35,132 (conscriptum molli et Xenophontio genere sermonis).

10. Cic. Brut. 35,132 (eo libro, quem...misit ad A.Furium poetam). Sui poemi di Archia cfr. Cic. Pro Archia 3,5.

11. Da Sonnabend 2002, p.95: «Sullas Hypomnemata stellen bei dieser Entwicklung einen Markstein dar...»; in precedenza cfr. soprattutto Lewis 1991 e Behr 1993, in seguito cfr. invece Scholz 2003.

12. Plutarco nella Vita di Silla cita sempre (otto volte) hypomnémata, Plut. Lucull. 1,3 parla di $\alpha$ v̇̃õ̃

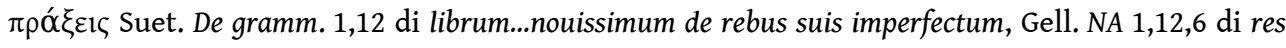
gestae, Priscian. 9,39 di res suae.

13. Sonnabend 2002, p.95-96 ha molto, forse troppo, insistito sul fatto che in Silla l'autobiografia evolve da rappresentazione apologetica a rappresentazione positiva proprio attraverso il tema del favorito dalla Fortuna e dagli dei.

14. Plut. Lucull. 1,3 = fr. 1 Peter.

15. Ruolo di Epicado: Suet. De gramm. 1,12.

16. Silla come fonte: Calabi 1950; cfr. anche Valgiglio 1975 e Pascucci 1975 rispettivamente. 
17. Cic. De diuin. 1,33,72 = fr.9 Peter: ut in Sullae scriptum historia uidemus; l'emendazione Sisennae, proposta dall'Aldina 1523, è certamente da respingere come facilior: qui Cicerone vuole qualificare i commentarii di Silla come historia (in patente contraddizione con l'opinione di Silla stesso in Plut. Lucull. 1,3) per promuoverne l'obiettività e contrapporla alla faziosità di Cesare da lui denunciata nel Brutus (cfr. infra nota 23).

18. Le composizioni poetiche sono, come è noto, il de consulatu suo e il De temporibus suis, il memoriale sono gli anékdota de consiliis suis rielaborati nel 44/43 (cfr. Zecchini 1996), la ricerca dello storico o, forse meglio, del biografo è attestata dalla $E p$. Ad fam. 5,26 a Lucceio del 56 (cfr. ora Salamon 2009).

19. Su Atacino e Bibaculo cfr. Blänsdorf (ed.) 1995, p.239 e 203, nonché Narducci 1989, p.567 e soprattutto Dobesch 2006; il carattere immediatamente celebrativo di questi poemi, quasi instant books, è, soprattutto per Bibaculo, una mia ipotesi.

20. Catull. Carm. 11,11-12. Sul carattere non ironico di questi versi mi sono già espresso altrove (Zecchini 1987, p.254) sulla scia di Granarolo 1958.

21. Sulle varie teorie moderne riguardanti la composizione del Bellum Gallicum cfr. Zecchini 1978, p.12 nota 11, a cui si aggiunga ora, per una datazione anno per anno, Cesa 2007.

22. Rambaud $1953=1966^{2}$.

23. Cic. Brut. 262. Che dietro i riconoscimenti formali il giudizio di Cicerone sui commentarii di Cesare sia negativo mi sembra emergere già dalla similitudine con la donna che si spoglia; l'analisi migliore del passo è, secondo me, in Brugnoli 1993.

24. Canfora 1993 (e già Canfora 1970), analizzato e discusso da Loreto (ed.) 2001, p.7-41 in un'ottima sintesi, che non esclude l'autenticità della lettera e riconferma il ruolo primario di Irzio nell'attingere alle carte di Cesare e organizzare il completamento dei suoi commentarii.

25. Suet. DJ 56,4 con le mie considerazioni in Zecchini 2001, p.106-107.

26. L'ipotesi, da ultimo, in Pallavisini 1974, ma cfr. Loreto (ed.) 2001, p.26-31 (almeno due relazioni-base e un redattore proveniente dallo stato maggiore cesariano).

27. Così, almeno, Strocchi 1996, ma cfr. Loreto (ed.) 2001, p.31-34 (forse un liberto del dittatore).

28. Zecchini 1978, cit. alla nota 21 ; contra Jehne 1997, p.51.

29. Pretestato: Suet. De gramm. et rhetor. 10,1. Estensione delle Storie di Sallustio e di Pollione: Zecchini 1982, p.1281-1286.

30. Cfr. Zucchelli 1976.

31. Cfr. da ultimo Ferrary 1997.

32. Cfr. Zecchini 2009.

33. Le migliori ricerche sulla giovinezza di Cesare restano quelle di Strasburger 1938 e 1968, dove si insiste giustamente sul carattere retrospettivo, aneddotico e, in ultima analisi, assai dubbio di molte tra le notizie su Cesare antecedenti il 63.

34. Si badi che a Oppio Suetonio (DJ 56,19) attribuiva, sia pure in forma dubitativa, i tria bella, che completano il corpus Caesarianum, e di recente un'acuta suggestione di Loreto (ed.) 2001, p.39 indica in Oppio uno dei possibili, se non il più probabile, erede di Irzio nella redazione dello stesso corpus.

35. Le Imagines sono di solito datate verso il 39 e il De viris inlustribus di Nepote verso il 35/32 (su di esso cfr. Geiger 1985 e Tuplin 2000); si pensi anche al De familiis Troianis dello stesso Varrone e alle richieste di biografie gentilizie, a cui T.Pomponio Attico fu sottoposto almeno da parte di Bruto, Metello Scipione e del Marcello cos.50 e a cui corrispose componendo diversi libri sui Giunii, i Claudii Marcelli, i Cornelii Scipioni, i Fabii e gli Emilii (Corn.Nep. Vita Attici 18,3). Sull'attività biografica di Attico cfr. Marshall 1993.

36. Perciò, in quanto biografie singole e non sillogi di biografie, non compaiono nel noto elenco ieronimiano (contenuto nella praefatio del De viris inlustribus: de uiris inlustribus scripserunt apud Latinos Varro, Santra, Nepos, Hyginus et Suetonius) e perciò non è rilevante in questa sede la loro 
cronologia rispetto a tali sillogi; per l'eventuale anteriorità di Nepote nei confronti di Oppio cfr. Geiger 1985, p.83-84.

37. Un precedente della biografia di Varrone su Pompeo in tre libri (a noi nota solo dal catalogo ieronimiano) può forse rinvenirsi nei molti libri dedicati alle res gestae dei due Pompei, lo Strabone e il Magno, dal liberto di quest'ultimo C.Voltacilio Pitolao, ma la definizione di historia, che Suetonio dà a quest'opera (De gramm. 27), rende insicura la definizione del suo genere.

38. Su questa evoluzione cfr. Zecchini 1980.

39. Liu. 38,56 .

40. L'unico frammento di Cotta (1 Peter) si legge in Athen. 6,273b; commento in Zecchini 1995, p. 601-602.

41. E' nota la presentazione della guerra civile del 49 come una dignitatis contentio in Q.Aelii Tuberon. fr.4 Malcovati apud Quintil. I.O. 11,1,80; per la discendenza di Scipione da Giove cfr. Opp. fr.2 Peter = Aul.Gell. NA 6,1,1.

42. Il rancore di Cassio verso Cesare è denunciato da Plutarco (Brut. 8,5-7); cfr. anche App. B.C. 2,16,111-113.

43. Sid.Apollin. Ep. 9,14,7 a Burgundione del 477.

44. Cfr. rispettivamente Opp. fr.5 Peter $=$ Plut. Pomp. 10; fr.6 Peter = Plut. Caes. 17 ; fr.7 Peter $=$ Suet. DJ 53; Plut. Caes. 17 = Suet. DJ 72.

45. Sall. Catil. 54,3.

46. Sul 'pompeianesimo' di Livio cfr. da ultimo Mineo 2009.

47. Sall. Catil. 43,1, su cui cfr. Zecchini 1996.

48. Basti qui il rinvio a Brunt 1965.

49. Brevemente ora Sonnabend 2002, p.119-124.

50. Cfr. ora Pittia 2009.

\section{RIASSUNTI}

L'article examine autour de César, sa personne et ses écrits, les diverses modalités historiographiques à l'œuvre pour rendre compte de sa carrière très particulière. Il replace les écrits de César et sur César dans le contexte de la naissance progressive d'une historiographie littéraire à Rome et montre comment la figure du restaurateur a pu s'imposer pour un long moment face à celle du tyran, en raison même de la mainmise de la tradition césarienne sur l'historiographie de la période. 


\section{INDICE}

Mots-clés : autobiographie, biographie, commentarii, épistolographie antique, guerre civile romaine, histoire gentilice, histoire et épopée, histoire et poésie, historiographie romaine, littérature et politique, objectivité de l'historien, pratique éditoriale antique et médiévale, sacrilège, tyrannicide

Indice geografico : Gaule

Keywords : autobiography, biography, commentarii, ancient epistolography, Roman civil war, Roman family history, history and epic, history and poetry, Roman historiography, literature and politics, objectivity in history, ancient and medieval editorial practice, sacrilege, tyrannicide nomsmotscles Aelius Tubéron, Aemilius Scaurus, Appien, Archias, Arunculeius Cotta, Asinius Pollion, Athénée, Aulu-Gelle, Aulus Furius, César, Cicéron, Cornelius Epicadus, Dion Cassius, Furius Bibaculus, Hirtius (Aulus), Julius Maratus, Lutatius Catulus, Munatius Rufus, Nicolas de Damas, Oppius, Plutarque, Priscien, Rutilius Rufus, Salluste, Sidoine Apollinaire, Sisenna (Lucius Cornelius), Suétone, Sulla, Tite-Live, Varron d'Atax 